\title{
Evaluation of online continuing medical education regarding weight management for fertility and pregnancy: a mixed-methods study
}

\author{
R. Walker ${ }^{1}$, C. Bennett ${ }^{1}$, A. Kumar ${ }^{2}$, M. Adamski ${ }^{1}$, M. Blumfield ${ }^{1}$ and H. Truby ${ }^{1}$ \\ ${ }^{1}$ Department of Nutrition, Dietetics and Food, School of Clinical Sciences, Monash University, Notting Hill, Victoria, \\ Australia. and ${ }^{2}$ Department of Obstetrics and Gynaecology, School of Clinical Sciences, Monash University, \\ 246 Clayton Road, Clayton, VIC 3168, Australia.
}

Increasing rates of maternal overweight and obesity are strongly associated with suboptimal pregnancy and neonatal outcomes ${ }^{(1)}$. Health professionals report being ill-equipped to address the sensitive topic of weight management for pregnancy despite guidelines that advocate the importance of further training in this area ${ }^{(2,3)}$. Online continuing medical education (CME) has the potential to improve the efficiency and effectiveness of educational interventions ${ }^{(4)}$. Therefore, the aim of this study was to evaluate changes in health professionals' attitudes, knowledge and practice regarding weight management for fertility and pregnancy after completing the online CME entitled Food as Medicine: Food, Fertility and Pregnancy.

A mixed-methods study surveyed participants before $(n=106)$ and after $(n=43)$ completing two hours of online CME regarding weight management for fertility and pregnancy developed by academics at Monash University, Australia. Evaluation was based on Kirkpatrick's/Barr's Hierarchy ${ }^{(5)}$. Paired non-parametric data $(\mathrm{n}=26)$ assessed changes in attitudes and knowledge using McNemars and Wilcoxon Signed Rank tests $(p<0 \cdot 05)$. Qualitative data collection was embedded into a predominantly quantitative design to gain a deeper understanding of the impact of the CME. Deductive content analysis was used to explore how participants intended to change their practice $(n=43)$. Ethics approval was granted by Monash University (REF: 2016-0379).

Participants $(95.3 \%)$ reported that they were satisfied to highly satisfied with the online CME. They increased in confidence to identify women who would benefit from specialist dietary advice $(p=0.04)$ and to discuss weight management issues with women for pregnancy $(p<0.01)$. There was no change reported in participants' knowledge of gestational weight gain guidelines. Qualitative analyses revealed that participants intended to practice aspects of effective communication including, active listening, making eye-contact, showing empathy, and asking permission to discuss the sensitive topic of weight.

Food as Medicine: Food, Fertility and Pregnancy was well-received by participants, positively impacting attitudes and confidence. No change in knowledge was measured in the quantitative data, potentially due to the high level of knowledge of some participants before the course. Qualitative data indicated that participants acquired knowledge, confidence, or both, regarding effective communication strategies that may ultimately enhance how messages regarding weight management for pregnancy are received by women.

1. Cheney K, Farber R et al. (2018) Med J Aust 208, 119-125.

2. Heslehurst N, Russell S et al. (2012) Midwifery 29, 736-744.

3. Walker R, Kumar A et al. (2018) Nutr Bull 43, 69-78.

4. Lam-Antoniades M, Ratnapalan S et al. (2009) J Cont Educ Health Prof 29, 44-51.

5. Barr H, Hammick et al. (1999) Br Educ Res J 25, 533-44.

Supervisor: Professor Helen Truby, Department of Nutrition, Dietetics and Food, School of Clinical Sciences, Monash University, Notting Hill, Victoria, Australia.Helen.truby@monash.edu 